\title{
Application of LLR-BP optimization algorithm in MIMO-OFDM
}

\author{
Jia Huang ${ }^{1, a^{*}}$, Ruian Liu ${ }^{1, b}$, Lirong Diao ${ }^{1, \mathrm{c}}$ \\ ${ }^{1}$ Department of Electronics, College of Electronics and Communications Engineering, Tianjin \\ Normal University, TJ-300387 Tianjin, China, of first author, including country \\ adaleyhj@126.com, ruianliu@sina.com, ${ }^{\mathrm{c}} 1257243891 @ q q . c o m$
}

Keywords: LDPC, LLR-BP, MIMO, OFDM.

Abstract. This paper introduces the Multiple Input Multiple Output (MIMO), Orthogonal Frequency Division Multiplexing (OFDM) and Low Density Parity Check Code (LDPC). Then, this paper gives the improved log domain Back Propagation (BP) decoding algorithm optimizing the variable nodes and simplifying the check nodes. The ameliorate approach can reduce the decoding complexity, at the same time improving the decoding performance. Finally, builds a MIMO-OFDM communication system. Then it studies the application of LDPC on the MIMO-OFDM systems. Taking use the software simulation, this paper compares the influence of Bit Error Rate (BER) performance of different parameters on the system.

\section{Introduction}

MIMO technology is wireless communication systems based on the multiple-antenna, this technology can make wireless communication channel more efficient without increasing the bandwidth. In addition, MIMO can effectively convert the multipath effect into favorable factors and use them, multiplied improve the wireless channel capacity, spectral efficiency and data transmission rate $^{[1]}$. It also can improve the stability and performance of the communication system. OFDM technology have long symbol period. Therefore, it can effectively improve the impact caused by frequency selective fading, and reduce the inter-symbol interference. The MIMO technology combine with OFDM technology used in modern wireless communication systems, will play a significant role in the development of wireless communication field. LDPC with superior encoding performance in MIMO-OFDM Systems become an ideal choice for a new generation wireless communications.

\section{LDPC-MIMO-OFDM System}

Multipath effect will cause signal fading, therefore it considered harmful factors. MIMO system can minimize the multipath effect, and can make good use of the respective multi-path components, thereby improving system performance. But MIMO systems are unable to improve the effects caused by frequency-selective fading ${ }^{[2]}$. OFDM technology can divide a transmission channel of the communication system into a number of subchannels, subchannels signal bandwidth is narrower than the bandwidth of the original channel; the original channel frequency selectivity acting on subchannels can be regarded as flat fading, thereby eliminating the effect of Inter Symbol Interference $(\text { ISI })^{[3]}$. Because of the orthogonality between sub-channels each, OFDM can transform high-speed serial data stream into low-speed parallel sub-data stream, and the quadrature signal separation can be realized at the receiving side, which can further reduced Inter-Carrier Interference (ICI) ${ }^{[4]}$.

MIMO-OFDM system is combine MIMO and OFDM, data transmission rate can be highly raised by spatial multiplexing; Space-time diversity and OFDM make communication system reliability and availability of spectrum resources significantly improved ${ }^{[5]}$. Applying error correcting coding in MIMO-OFDM system can improve system reliability and anti-interference ability. MIMO-OFDM with superior performance LDPC become an ideal choice for wireless communications. 


\section{Optimization of LLR-BP Algorithm}

BP algorithm based on LDPC codes for Tanner graph structure. In the iterative process, reliability of information passes through the graph edges between nodes. The values obtained through a number of iterations tends to be stable, optimal decoding decisions accordingly. BP algorithm is used in LDPC codes, there are more close to the Shannon capacity limit performance. The main idea of BP algorithm is the transmission of information in the Tanner graph through updating of information between variable nodes and check nodes with the preset iterations.

The complexity of the algorithm can greatly reduce, if using the likelihood ratio to represent the transmission information that between nodes. The algorithm is called logarithmic domain likelihood ratio belief propagation algorithm, referred to log-domain BP algorithm or LLR-BP algorithm.

\subsection{Optimization of Variable Message}

BP decoding algorithm use received information to iterative calculation in check node and variable node of the Tanner graph. Variable nodes collect useful message and make a judgment ${ }^{[6]}$. In the Tanner graph acyclic or substantially acyclic, variable node message can converge until a posterior probability while iterative decoding number tends to infinity.

However, in practice applications, limit of LDPC codes length causes the Tanner graph with a loop exists, a shorter loop the decoding of LDPC codes has a significant performance impact. Therefore, This paper introduces a multiplicative weakening related factor basis on LLR-BP algorithm that can compensated variable correlation between the messages in the process variable message.

Variable node messages processing of LLR-BP optimization algorithm is added a related factor of multiplicative weakening $\mu \in(0,1)$

$$
\operatorname{LLR}^{\prime}\left(q_{i j}\right)=\ln \frac{q_{i j}(\mathrm{O})}{q_{i j}(1)}=\mu \cdot\left(\operatorname{LLR}\left(p_{i}\right)+\sum_{\tilde{i} \in M(j) \backslash i}^{n} \operatorname{LLR}\left(r_{\tilde{i} j}\right)\right)
$$

on the basis of LLR-BP algorithm $L L R\left(q_{i j}\right)=\ln \frac{q_{i j}(0)}{q_{i j}(1)}=\operatorname{LLR}\left(p_{i}\right)+\sum_{\tilde{i} \in M(j) \wedge i}^{n} L L R\left(r_{\tilde{i} j}\right) \cdot$ related factor of Multiplicative weakening need to determine by simulation. The algorithm adds a multiplication operation to the iteration and the computational complexity of the algorithm does not increase.

\subsection{Simplified Check Node}

Simplify validation information updating process of exponential, logarithmic and multiplication that can reduce the computational complexity of the decoding of each check (variable) node in LLR-BP algorithm. Therefore, simplified check nodes is an effective method to reduce LLR-BP complexity $^{[7]}$.

$\phi(x)$ is a known transfer function:

$$
\phi(x)=-\ln \left(\tanh \left(\frac{x}{2}\right)\right)=\ln \left(\frac{e^{x}+1}{e^{x}-1}\right)
$$

As we know, $\phi(x)$ have large function values while the independent variables small. $\phi(x)$ increases rapidly decay when the independent variables. Function $\phi(x)$ has the following two properties:

$$
\begin{aligned}
& \phi(\phi(x))=x \\
& \phi^{-1}(x)=\phi(x)
\end{aligned}
$$

In the LLR-BP decoding, the check node calculation can be expressed as:

$$
\left.\operatorname{LLR}^{(l)}\left(r_{i j}\right)=2 \tanh ^{-1}\left(\prod_{\tilde{j} \in N(i)\rangle_{j}} \tanh \left(\frac{1}{2} L L R^{(l-1)}\left(q_{i j}\right)\right)\right)=\prod_{\tilde{j} \in N(i) j_{j}} \operatorname{sgn}\left(L L R^{(l-1)}\left(q_{i j}\right)\right) \times \phi\left(\sum_{\tilde{j} \in N(i)\rangle_{j}} \phi \mid L L R^{(l-1)}\left(q_{i j}\right)\right)\right)
$$

$(l)$ is the number of iterations.

Based on the analysis nature of function, simplify the update process of check message:

(1) When $\min _{\tilde{j} \in N(i)\rangle_{j}} \mid L L R^{(l-1)}\left(q_{i j}\right)>\lambda$, check nodes messages will be updated as follows: 


$$
\operatorname{LLR}^{(l)}\left(r_{i j}\right)=\prod_{\tilde{j} \in N(i) \backslash j} \operatorname{sgn}\left(L L R^{(l-1)}\left(q_{i j}\right)\right) \times \min _{\tilde{j} \in N(i)\rangle_{j}}\left|L L R^{(l-1)}\left(q_{i j}\right)\right|
$$

$\lambda$ is a certain value.

(2)When $\min _{\tilde{j} \in N(i) \backslash j}\left|\operatorname{LLR} \boldsymbol{R}^{(l-1)}\left(q_{i j}\right)\right|<\lambda$, a degree d check nodes degree $\mathrm{d}$ will sort $\left|L L R^{(l-1)}\left(q_{i j}\right)\right|(\widetilde{j} \in N(i) \backslash j)$ as order of small to large. Place the smallest q $\tilde{j}$ of $\mid L L R^{(l-1)}\left(q_{i j}\right)(\tilde{j} \in N(i) \backslash j)$ into set $N_{q}, 2 \leq q \leq d-1$. Check nodes messages update as:

$$
L L R^{(l)}\left(r_{i j}\right)=\prod_{\tilde{j} \in N(i) \backslash j} \operatorname{sgn}\left(L L R^{(l-1)}\left(q_{i j}\right)\right) \times \phi\left(\sum_{\tilde{j} \in N_{q}(i) \backslash j} \phi \mid L L R^{(l-1)}\left(q_{i j}\right)\right)
$$

In calculating the updated of check nodes messages, the minimum and the second minimum of argument nodes messages' absolute value have the greatest impact on reliable information ${ }^{[8]} . \lambda$ is selected by computing similar parameter value of rapid convergence algorithm in Literature [9], therefore $\lambda=5.3$.

According to this two methods, the computational of improved algorithm is less complexity than LLR-BP. Moreover, the decoding performance of improved algorithm is almost consistent with LLR-BP .

\section{Simulation Analysis}

Simulation analysis LDPC decoding algorithm impact on system performance based on different factors in LDPC MIMO-OFDM system. This paper compares the BER performance of the system. The basic parameters are set as follows: Simulation experiments use irregular QC-LDPC codes, code length $n=1010$ bit, code rate $\mathrm{R}=1 / 2$, coding method based on Gaussian elimination, decoding algorithm using LLR-BP algorithm and improved algorithm, decoding iterations number is 50 . Rayleigh fading channel, multipath number is 6.

\subsection{Different Iterations Effect on the System}

LDPC code has a sparse parity check matrix $\mathrm{H}$, so that it has the characteristics of random code. Iterative decoding algorithm made a longer LDPC codes length. These two factors also make LDPC codes have excellent error correction performance channel. This paper use different iterative number of LLR-BP to simulation.

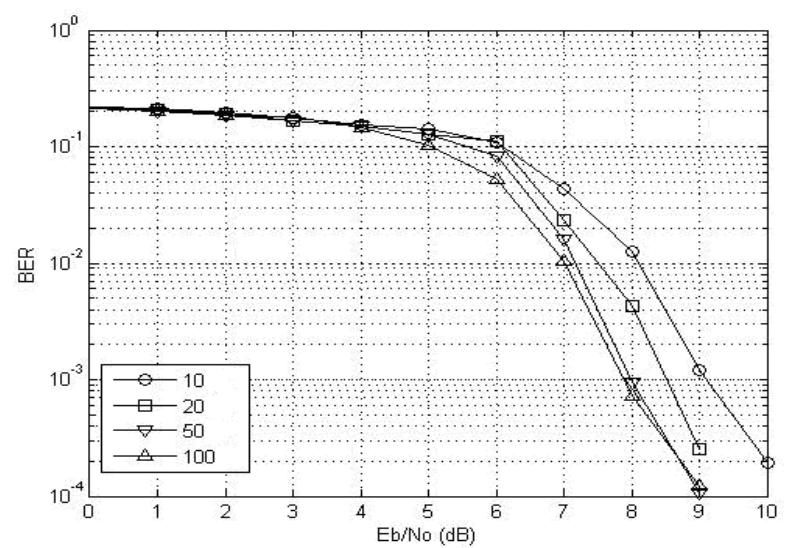

Fig.1. Effect of Different Iterations to the System

As can be seen in Fig.1, with the increasing number of iterative decoding, system error rate gradually decreased, and the larger number the faster convergence. A "waterfall area" appear Between SNR $7 \sim 9 \mathrm{Db}$. The number of decoding algorithm iteration have a great impact on the performance of LDPC decoding. However, when the number of iterations large enough, widely increases the number of iterations have no obvious improvement on the BER performance, such as compare the result of 50 iterations with 100 . Therefore, we can not blindly increase the number of iterations to improve system performance, besides, increasing the number of iterations will increase decoding time, which resulted in reducing the effectiveness of communication system. 


\subsection{Different Modulation Mode Effect on the System}

Transmitted messages by LDPC encoding required constellation mapping, different modulation mapping will have a direct impact on LDPC-MIMO-OFDM. Figure 5 shows BER performance while the modulation scheme is BPSK, QPSK, 8PSK, 16PSK and 32PSK.

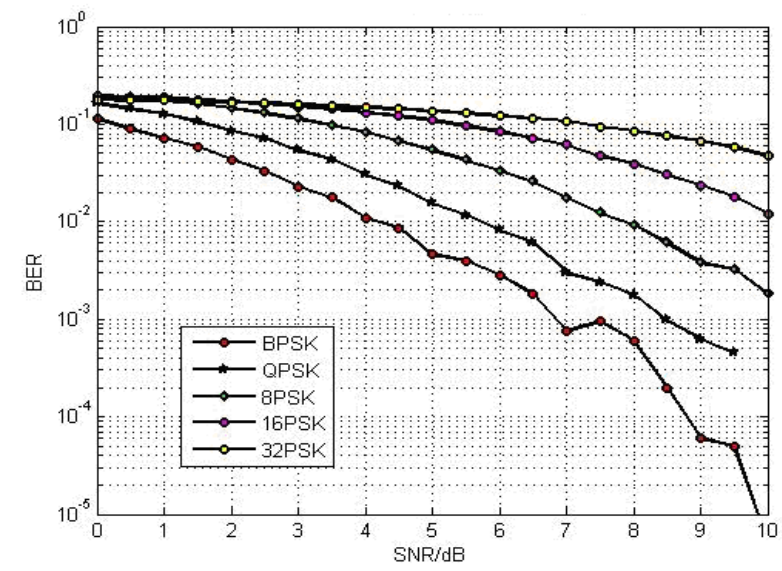

Fig.2. Comparison of Different Modulation

In Fig.2, with gradually increases of hexadecimal number, BER performance of the system is getting worse. Besides, BPSK get optimal performance, 32PSK get the worst performance. With the hexadecimal number increasing, the difference between message symbols become small. Therefore, decision is more difficult when demodulating. With the decrease of hexadecimal number, bandwidth efficiency is reduced, which resulted in wasting of spectrum resources. Therefore, modulation is important to LDPC-MIMO-OFDM system.

\subsection{Different Antenna Number Effect on the System}

Multi-antenna system resist fading by using multiple antennas transmit and receive diversity. Therefore, the number of transmit and receive antenna is a main factor affecting the channel capacity and reliability of the system.

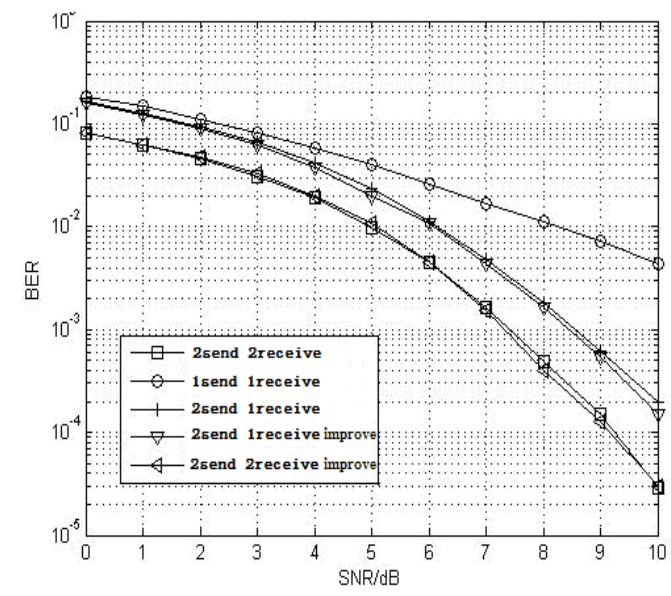

Fig.3. Comparison of Different Antenna Number

This experimental simulations using multiple transmitting and receiving antennas. As can be seen in Fig.3, using 2 transmit 2 receive antenna works best, the improved algorithm improved BER 0.05 0.1 dB during SNR 7 8dB, but improved algorithm do not have a obvious performance improve at other SNR.

\subsection{The Number of OFDM Subcarriers Effect on the System}

As can be seen from Fig.4, the solid line shows that LDPC-MIMO-OFDM system BER performance is reduced while the number of OFDM subcarriers is gradually increased. This is due to the increased number of subcarriers, the high speed transmission data stream turn into lower speed data streams. Therefore system anti-frequency selective fading performance have effectively improved so that BER performance has been gradually improved. 
The dotted line results is the improved algorithm. Due to LDPC-MIMO-OFDM high complexity of the system itself, and channel for Rayleigh fading channel, the improved algorithm have less contribution to resistance to frequency selective fading while the data stream speed becoming low, the improved performance was not obvious. Improved decoding performance improvement were maintained at an average level. In the case of a different number of subcarriers, improving decoding algorithm improve the system performance about $0.1 \mathrm{~dB}$ whileBER $=10^{-4}$.

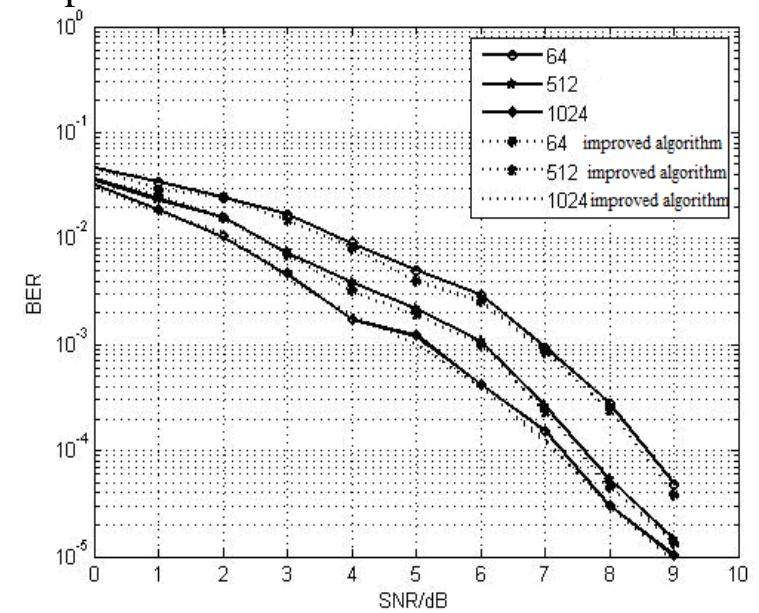

Fig.4. Compare the Number of OFDM Subcarriers

\section{Summary}

This paper firstly introduces LDPC-MIMO-OFDM system, and analyzes the theoretical basis of the iterative decoding algorithm. Then this paper optimizes the LLR-BP algorithm. Simulation analyze system performance in case of different variable arguments. Therefore the improved decoding algorithm do not have a obviously BER improvement of LDPC-MIMO-OFDM system Improved decoding algorithms do not damage to the system's performance, but there is a stable tendency.

\section{References:}

[1] Tao Huang, Chaowei Yuan, Ruizhe Yang. MIMO-related Technology and Applications[M]. eijing: National Defence Industry Press, 2006:39-77.

[2] S.A. Mujtaba. MIMO Signal Processing: The Next Frontier for Capacity Enhancement[J], IEEE Custom Integrated Circuits Conference, Vol.1, 2003, pp 263-270.

[3]K.R.Venugopal, L.M.Patnaik. Inter-Carrier Interference Power Analysis of OFDM Systems under Slowly Fading Channels[D]. Wireless Personal Communications , 2012, pp 11-17.

[4] S. Jamallulil, J. P. Cances, V. Meghdadi. Space-Time-Block-Coded Transmissions for Downlink MC-CDMA Transmissions Over Frequency-Selective Fading Channels. Wireless Personal Communications , 2006, Volume 39, Issue 1, 63-79.

[5] Lu Zhaogan, Rao Yuan, Zhang Taiyi, Wang Liejun. Multiuser MIMO OFDM Based TDD/TDMA for Next Generation Wireless Communication Systems[J]. Wireless Personal Communications, 2010, Volume 52, Issue 2, pp 289-324.

[6]Chutima Prasartkaew, Somsak Choomchuay. A Design of Parity Check Matrix for Irregular LDPC Codes[C]. 9th International Symposiun on Communications and Information Technology. 2009, 9: 239-242.

[7] Wang Ying, Xie lei. Improved decoding algorithm of bit interleaved coded modulation for LDPC code [J]. IEEE Transactions on broadcasting. 2010, 56(1): 103-109.

[8]LiJun Zhang, Bing Li, LeeLung Cheng. Constructions of QC LDPC codes based on integer sequences[J]. Science China Information Sciences, 2014, Volume 57, Issue 6 , pp 1-14 .

[9] Yang Xiao. Turbo and LDPC codec and its application[M]. Beijing: People's Posts and 
Telecommunications Press, 2010:38-71.

\section{Acknowledgments}

This research was supported by the Tianjin natural science. fund(13JCYBJC15800) 